\title{
THE SAFAR CENTER FOR RESUSCITATION RESEARCH: SEARCHING FOR BREAKTHROUGHS IN THE NEW MILLENNIUM
}

\author{
P. M. Kochanek ${ }^{1}$, H. Bayir ${ }^{1}$, R. P. Berger², C. E. Dixon³ ${ }^{3}$ L. Jenkins ${ }^{3}$, A. E. Kline ${ }^{4}$, \\ S. Tisherman ${ }^{5}$, A. K. Wagner ${ }^{4}$, R. S. B. Clark ${ }^{1}$ \\ ${ }^{1}$ Department of Critical Care Medicine University of Pittsburgh School of Medicine Pittsburgh, Pennsylvania, USA \\ ${ }^{2}$ Department of Pediatrics Children's Hospital of Pittsburgh Pittsburgh, Pennsylvania, USA \\ ${ }^{3}$ Department of Neurological Surgery University of Pittsburgh School of Medicine Pittsburgh, Pennsylvania, USA \\ ${ }^{4}$ Department of Neurological Surgery University of Pittsburgh School of Medicine Pittsburgh, Pennsylvania, USA \\ ${ }^{5}$ Departments of Surgery and Critical Care Medicine University of Pittsburgh School of Medicine Pittsburgh, \\ Pennsylvania, USA
}

\begin{abstract}
This review, written on the occasion of the $\mathbf{7 0}^{\text {th }}$ anniversary of the Institute for General Reanimatology of the Russian Academy of Medical Sciences, provides an update of recent research in the field of resuscitation medicine carried out at the Safar Center for Resuscitation Research at the University of Pittsburgh School of Medicine. Current and recent studies describing bench to bedside investigation in the areas of traumatic brain injury (TBI), cardiopulmonary arrest, hemorrhagic shock, and ultranovel approaches to resuscitation are discussed. Investigation in TBI across a variety of topics by many investigators including mechanism of neuronal death, oxidative and nitrative stress, proteomics, adenosine, serotonin, novel magnetic resonance imaging application, inflicted childhood neurotrauma, and TBI rehabilitation is addressed. Research discussed in the program of cardiopulmonary arrest includes optimization of the use of mild hypothermia and novel investigation in experimental asphyxial cardiac arrest. In the program on hemorrhagic shock, our recent work on the application of mild hypothermia to prolong the «golden hour» is presented. Finally, a brief overview of our studies of a novel approach to the resuscitation of exsanguination cardiac arrest using emergency preservation for resuscitation (EPR) is provided. Key Words: Traumatic Brain Injury, Resuscitation, Child Abuse, Inflicted Childhood Neurotrauma, Hemorrhagic Shock, Emergency Preservation and Resuscitation, Apoptosis, Adenosine, Proteomics.
\end{abstract}

\section{Introduction}

First, let me congratulate Dr. Victor Moroz on the occasion of the the $70^{\text {th }}$ anniversary of the Institute for General Reanimatology of the Russian Academy of Medical Sciences. It is a truly amazing accomplishment and one that I know would have had special meaning to the late Dr. Peter Safar. There is a rich and special history of interaction between the late Drs. Peter Safar and Dr. Vladimir Negovsky, two giants in the history of resuscitation. To commemorate your anniversary celebration, I have provided this review article, which presents a very brief historical overview of the interface between Drs. Safar and Negovsky, followed by a description of the evolution of the International Resuscitation Research Center (IRRC) at the University of Pittsburgh School of Medicine into the Safar Center for Resuscitation Research-including an overview of some of our current programs along with a selection of interesting recent findings from our center.

The Safar-Negovsky Legacy

On September $5^{\text {th }} 1962$, Drs. Peter Safar chaired a panel discussion at the First European Anesthesiology Congress in Vienna, Austria on the topic of «Controversial aspects of resuscitation». Despite the challenges imposed by the cold war in the early 1960s, Dr. Safar invited Dr. Vladimir Negovsky to participate [1]-having read one of the English translated versions of Dr. Negovsky's work. That meeting began a lifelong friendship and mutual admiration between the two individuals who arguably became the most important figures in the history of modern resus- citation. Dr. Safar was impressed by four seminal aspects of the pioneering work of Dr. Negovsky [2-4], namely 1) his establishing a resuscitation research center as early as 1937, 2) his early documentation of the use of centripetal intra-arterial blood pumping and mechanical ventilation to resuscitate that was initially suggested by Russian physician Andreev in 1913, 3) his coining of the term «reanimatology» and 4) his description of the concept of «post-resuscitation disease». Dr. Safar's own pioneering work in resuscitation (reviewed in 5) included the development of mouthto-mouth resuscitation, the assembly and dissemination of CPR, his work on the application of mild hypothermia in cerebral resuscitation, his key role in the development of emergency medicine services for field resuscitation (which also included design of the modern cabin configuration of the ambulance), seminal studies on the pathomechanisms of human death in natural and man-made disasters, and ultimately, his spearheading the development of novel approaches to resuscitation such as emergency preservation and resuscitation (EPR). I believe that the mutual respect and sharing of ideas between these two colleagues influenced Negovsky to become more treatment oriented and Safar to further study pathophysiologic mechanisms [1] (Figure 1). The passing of these two giants and great leaders in resuscitation - both during the first week in August 2003 - represented a huge loss for the resuscitation community. However, their work has provided us with an incredibly rich foundation upon which new approaches can be developed to «save hearts and brains too good to die». 


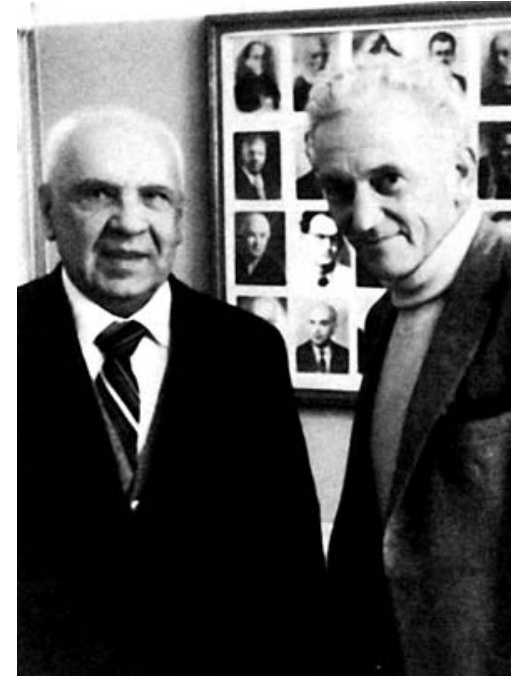

Figure 1. Vladimir Negovsky, «the father of reanimatology» and Peter Safar in Moscow in the 1970s.

Transition from the IRRC to the Safar Center In 1994, Dr. Peter Safar stepped down as director of the IRRC, to focus his efforts more on his research and a number of other projects. At that time, Dr. Safar had established research programs at the IRRC in the areas of cardiopulmonary arrest, hemorrhagic shock, and disaster medicine. I had the good fortune of being named director after a local competition between three faculty investigators in the Department of Anesthesiology and Critical Care Medicine at the University of Pittsburgh. Over the past 12 years, several new programs have been developed at the center and the landscape of several of the existing programs has evolved. Currently, we have programs in traumatic brain injury (TBI), cardiopulmonary arrest, hemorrhagic shock, and ultraadvanced resuscitation approaches such as EPR. Several important new investigators have been added to the center, and a number of key collaborators are involved. New and/or enhanced roles for molecular biology, free radical biology, proteomics, CNS injury models, and rehabilitation research have emerged. Our center has a multidisciplinary faculty which spans the departments of Critical Care Medicine, Neurological Surgery, Pediatrics, Surgery, Physical Medicine and Rehabilitation, Emergency Medicine, Anesthesiology, and Pathology. A synthesis of some of the most exciting and important findings within each of these programs is provided below. Finally, one former program is currently inactive, and that is the program in disaster medicine.

\section{Current Investigation at the Safar Center for}

\section{Resuscitation Research}

Traumatic Brain Injury: Bench to bedside

This represents a new area of research that has developed during the past 10 years. It has been the most productive area of investigation in our center and includes work -as principal investigator-by eight faculty investigators, and numerous important collaborators and trainees. Currents studies in our center are focused on mechanisms in the evolution of secondary damage including delayed neuronal death, oxidative and nitrative stress, dopamine systems, poly-ADP ribose polymerase (PARP), kinase- and phosphatase-mediated events, serotonin pathways, and endogenous neuroprotectants such as adenosine. We and our collaborators have taken advantage of a number of important new tools in this work on TBI, including the use of a contemporary model of experimental TBI-controlled cortical impact (CCI), the application of novel magnetic resonance imaging (MRI) tools such as perfusion imaging and non-invasive assessment of macrophage accumulation in injured brain, novel proteomic and oxidative lipidomic approaches, and in vivo electrochemical monitoring techniques like fast scan cyclic voltammetry, among others methods. We have used the CCI model of TBI in both rats in mice for over sixty studies in experimental TBI. This model was developed for use in rats by Dr. C. Edward Dixon, one of the Safar Center faculty [6], and is both highly reproducible, and well characterized. In addition, we have developed several modifications of this model, most notably the use of combined TBI plus secondary hypoxemia [7]-which models the important combination of these insults that is often observed clinically after TBI [8]. Finally, our group has led the way in linking bench-bedside in the study of cellular and molecular mechanism of secondary damage and repair in human TBI. We have accomplished this using two important resources, assessing biochemical and molecular markers in cerebrospinal fluid (CSF) drained through ventriculostomy catheters in the treatment of intracranial hypertension in patients (both adults and children) with severe TBI [reviewed in 9], and by performing western analysis and immunohistochemistry of brain tissue resected emergently from critically ill patients with severe TBI in the treatment of refractory intracranial hypertension and impending herniation [10-12].

\section{Mechanism of neuronal death}

This area of investigation has been the focus of Safar Center investigator, Dr. Robert Clark-who has contributed a number of important reports in both experimental and clinical TBI. Dr. Clark's most significant contributions in this area have included the first documentation of caspase- 3 and caspase- 1 activation in human head injury [10] (Figure 2), and early descriptions of the role of both caspase activation and apoptosis-initiating factor (AIF) release in experimental TBI [13, 14]. Most recently Dr. Clark and his group have published two reports in human TBI that have substantially advanced our knowledge in this area. First, Zhang et al [12] reported that the important cell survival pathway linked to protein kinase B 

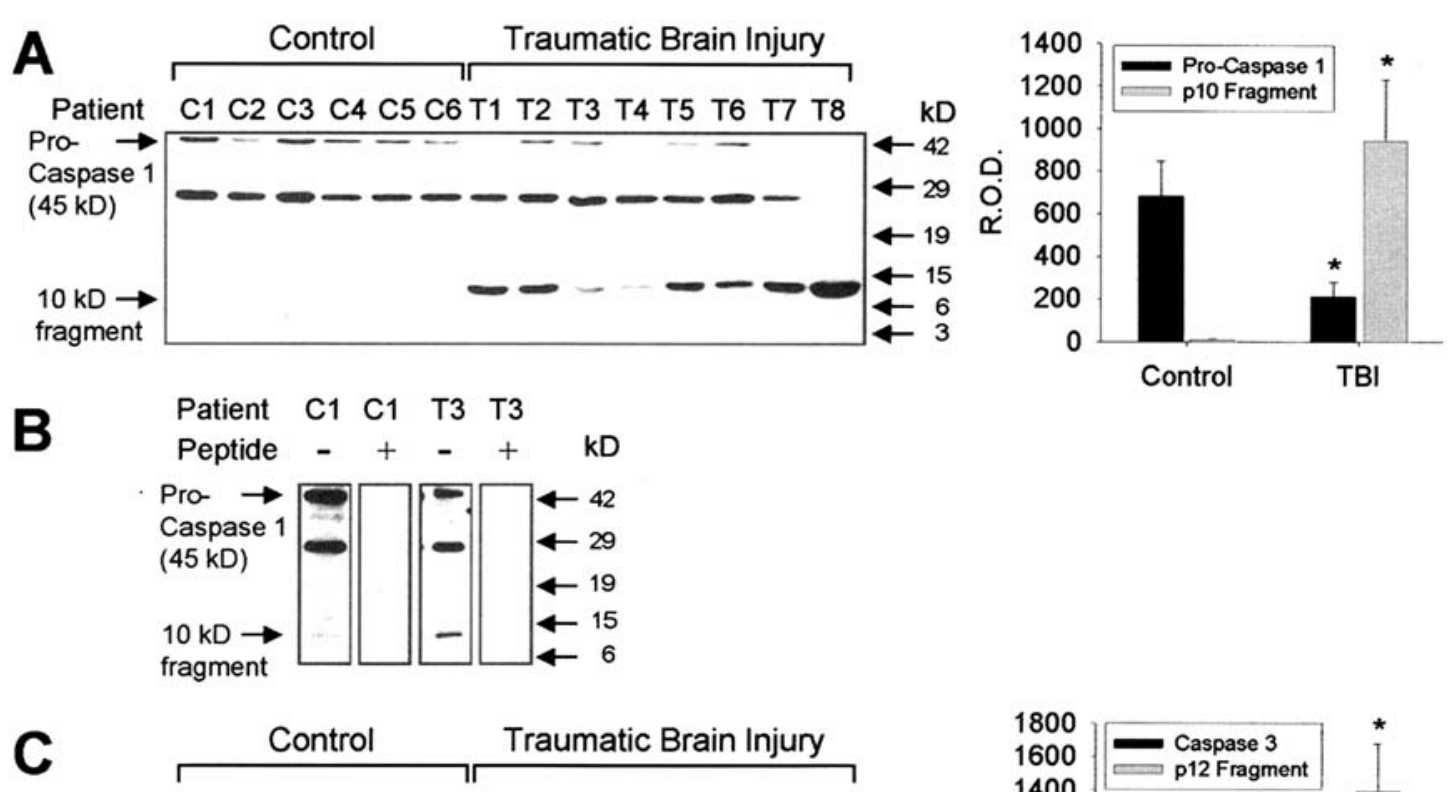

Patient C1 C2 C3 C4 C5 C6 T1 T2 T3 T4 T5 T6 T7 T8
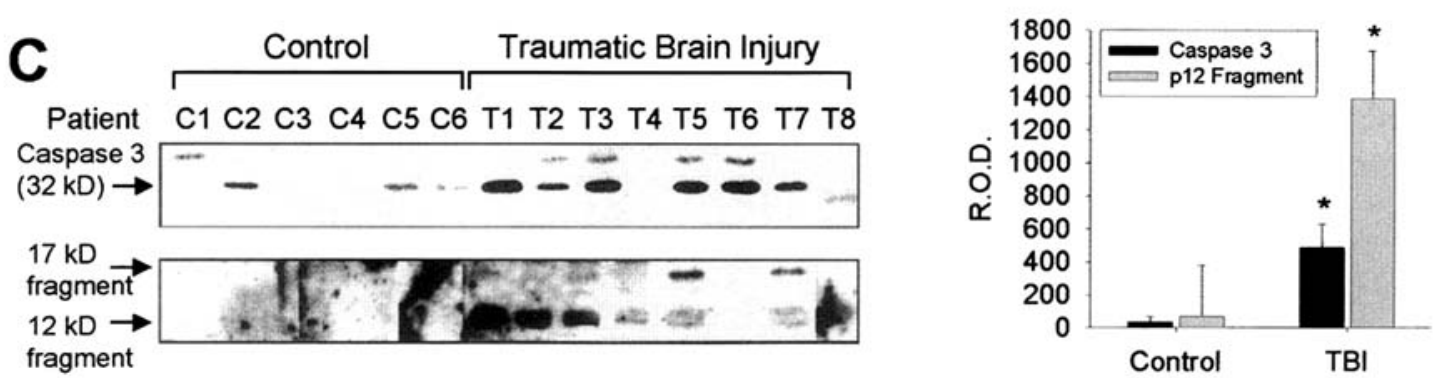

Figure 2. Example of bench to bedside molecular biology in human head injury from the laboratory of Dr. Robert Clark. Caspase-1 and -3 proteins are expressed in pericontusional brain samples from patients after severe traumatic brain injury (TBI). Caspase-1 is involved in synthesis of the pro-inflammatory cytokine interleukin-1, while caspase-3 is a critical enzyme in the apoptotic cell death cascade. A) Pro-caspase-1 was reduced in patients after TBI compared with controls. The p10 fragment of caspase-1 was increased in patients after TBI compared with controls. B) Preabsorption studies using the anti-caspase-1 p10 antibody incubated with or without the peptide used to generate the antibody. $C$ ) Both caspase-3 and the $\mathrm{p} 12$ fragment of caspase-3 were increased in patients after TBI versus controls. * $p<0.05$ vs. control, ROD, relative optical density. From Clark et al (10) with permission.

(PKB, also know as Akt) is activated in human TBI. PKB-when activated is phorphorylated and inhibits the intrinsic (mitochondrial) pathway of apoptosis. This is believed to be accomplished by PKB-mediated phosphorylation and inactivation of downstream death signals such as BAD and by phosphorylation and activation of the pro-survival signal cyclic AMP response element binding protein (CREB). Using brain tissue samples from 15 patients with severe TBI, Dr. Clark's group convincingly demonstrated that PKB and its downstream signals are phosphorylated and thus activated in human head injury-supporting a role for this important pathway and suggesting that it may be a target for therapeutic manipulation. These findings have taken on even greater significance given the recent work from the laboratory of Dr. Gary Steinberg suggesting that mild therapeutic hypothermia may confer some of its benefit through the enhancement of this pathway [15].

A second important recent publication from Dr. Clark's laboratory by Satchell et al [16], reported that levels of the key cellular apoptosis trigger cytochrome $\mathrm{C}$ are increased in CSF of infants and children with severe TBI-particularly in victims of inflicted childhood neurotrauma (i. e., child abuse or «shaken baby syndrome»). Because release of cytochrome $\mathrm{c}$ has been shown to be a pivotal trigger of the apoptotic cascade in a number of experimental systems, this study of 67 infants and children suggests that delayed neuronal death plays an important role in pediatric TBI, and that this pathway may be a particularly important therapeutic target in victims of child abuse. Targeting delayed neuronal death appears to represent an important therapeutic target for the development of new therapies for TBI, particularly in infants and young children.

Oxidative and nitrative stress

Dr. Hulya Bayir of the Safar Center, in collaboration with Dr. Valerian Kagan, at the University of Pittsburgh Center for Free Radical and Antioxidant Health, have carried out a series of studies over the past 5 years that has contributed significantly to our understanding of the role of oxidative and nitrative stress in experimental and clinical TBI [17-24]. Using a comprehensive battery of markers of oxidative stress, Bayir et al [16] reported marked increases in lipid peroxidation and depletion of endogenous antioxidants such as ascorbate and total antioxidant 


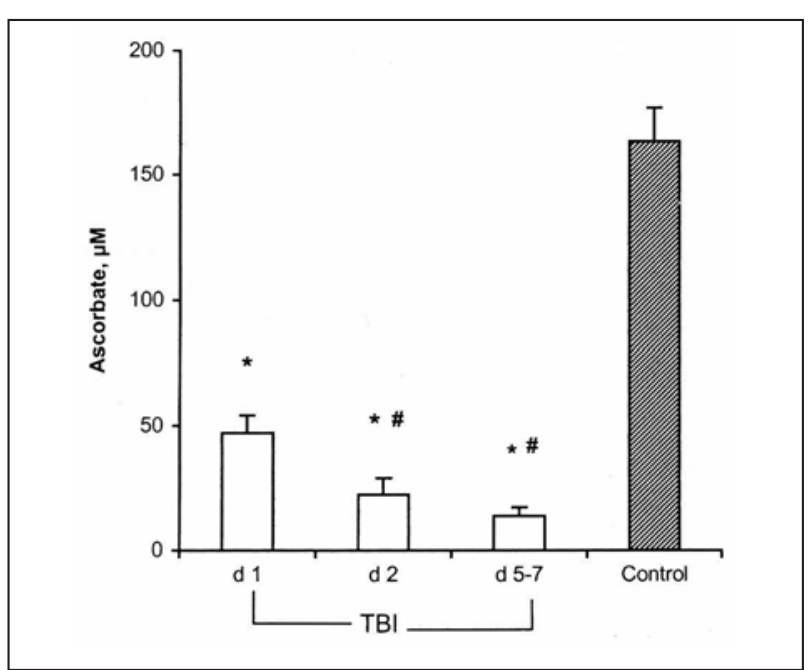

Figure 3. Effect of severe TBI on the antioxidant ascorbate concentration in CSF of infants and children. Ascorbate concentrations were 3-, 8-, and 10-fold lower in subjects with TBI on d 1, d 2 , and $\mathrm{d} 5-7$, respectively, compared with controls $\left({ }^{*}-p<0.05\right.$ vs control, \# $-p<0.05$ vs TBI d 1). These findings strongly support that the injured brain is depleted of endogenous antioxidants and may be vulnerable to secondary oxidative insults in the intensive care unit. From Bayir et al (10) with permission.

reserve in CSF samples in children with severe TBI. Striking and concerning was the extent and progressive nature of the ascorbate depletion in these patients over the initial 5 days after severe TBI (Figure 3). This report strongly suggests that one of the mechanisms underlying the well recognized heightened vulnerability of patients with severe TBI to secondary brain insults in the intensive care unit, reported by Gopinath et al [25], is loss of antioxidant defenses. More recently, in preliminary studies, Dr.

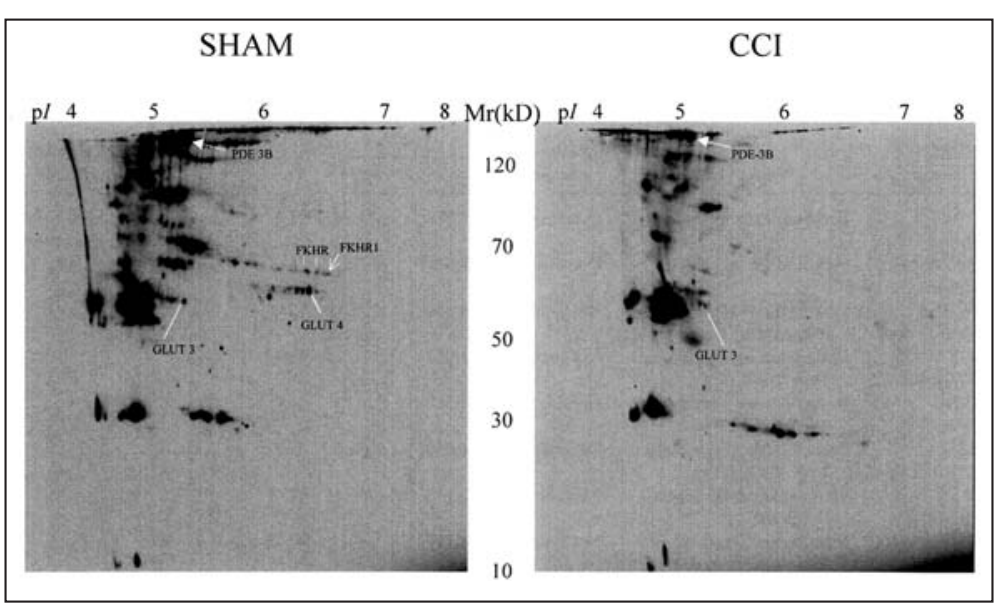

Figure 4. Proteomic assessment using large-format 2D immunoblots showing a reduction in the number of phosphoproteins after experimental TBI in rats (135 spots) versus sham rats ( 209 spots). In addition to such general changes, spot candidates for a number of known protein substrates, such as forkhead transcription factors (FKHR, FKHR1), phosphodiesterase-3-beta (PDE-3B), glucose transporter protein 3 (GLUT3), and glucose transporter protein 4 (GLUT4), were proposed on the basis of observed $\mathrm{Mr}$ and $\mathrm{pI}$ values. Comparable spot candidates for FKHR, and FKHR1 phospho-immunoreactivity were not seen after CCI, with considerable loss of phosphor-immunoreactivity in GLUT3 and GLUT4. This powerful proteomic method demonstrates the ability to simultaneously assess changes in multiple phosphorylated proteins after experimental TBI. From Jenkins et al (26) with permission.
Bayir - in collaboration with Dr. David Adelson and his recent randomized controlled clinical trial of the use of mild to moderate hypothermia in infants with severe TBI, demonstrated that cooling significantly attenuated the loss of antioxidants after TBI [22] uggesting that this mechanism accompanies the prestion in intracranial pressure that is known to be in clinical TBI. Finally, in recent studies in neuronal nitric oxide synthase knockout mice, that that MnSOD nitration and inactivation occur in tusion samples from humans with severe TBI [23]. This is another example of the type of bench-to-bedside work that we feel is important to enhancing our understanding of cellular and molecular mechanism of secondary injury in severe TBI. Additional research on the role of oxidative and nitrative stress

\section{Proteomics applications}

One of the best methods currently available to try to unravel the mechanism of secondary injury and repair in the injured brain after TBI or cardiac arrest 作 (he examination of critical protein post-translational modification such as phosphorylation (Figure 4). Dr. Larry Jenkins and his associates in our center have contributed the initial application of this contemporary technology to the field of experimental TBI [26], the first use of this method in a developmental TBI model [27], and early use in clinical TBI [28]. In a manuscript in press [27], Dr. Jenkins' group used a 2D difference gel electrophoresis (DIGE) method to study the response to experimental brain injury in the rat hippocampus at 14 days after CCI injury in developing (17 day-old) rats. Changes in a large number of important proteins across numerous functional categories such as energy metabolism and mitochondrial function, synaptic function, oxidative stress, and cell stress and chaperone proteins were identified. For example, 30\% decreases in pyruvate dehydrogenase E1 beta and alpha subunits were noted, while the glial stress protein GFAP was increased over 300-fold. More recently, Gao et al [28] used a 2D gel approach to study CSF from infants and children with 


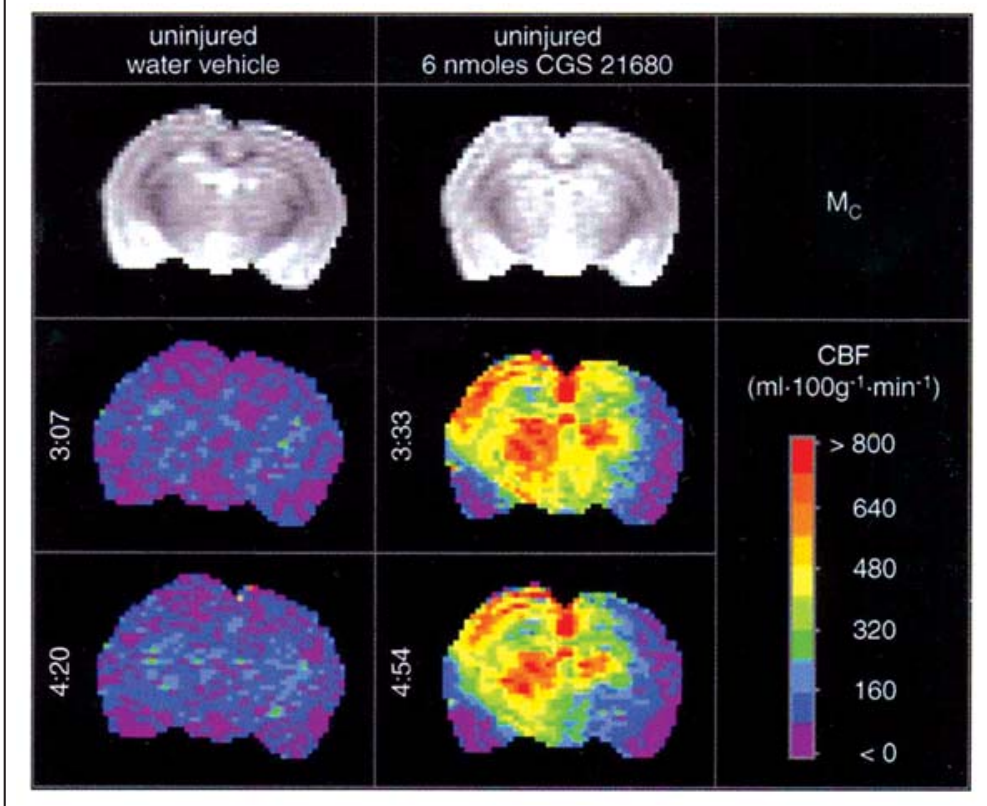

Figure 5. Acute effects of injection of either sterile water vehicle (left three vertical panels) or the selective adenosine $2 \mathrm{~A}$ receptor agonist, CGS 21680 (6 nmol, right three vertical panels) into the left dorsal hippocampus of uninjured rats on

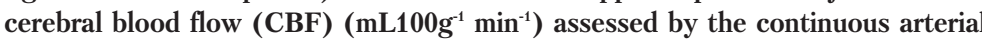
spin labeled magnetic resonance imaging (MRI) method. This contemporary MRI method is able to non-invasively demonstrate dramatic increases in CBF in rat brain by this adenosine A2a receptor agonist. From Kochanek et al (35) with permission.

either inflicted (child abuse) or non-inflicted (accidental) TBI. He demonstrated unique increases in the acute phase reactants such as haptoglobin isoforms in infants with accidental but not inflicted TBI. This suggests that factors such as a delay in presentation or chronic or repeated injury may blunt the traditional acute phase response in victims of TBI from child abuse.

\section{Adenosine}

Our group, in collaboration with Dr. Edwin Jackson of the University of Pittsburgh Center for Clinical Pharmacology, has carried out a number of studies demonstrating marked increased in levels of adenosine in brain interstitial fluid and CSF after both experimental and clinical TBI [29-32]. We have also characterized some of the putative effects of adenosine production in the injured brain, in both our model systems and in humans [31, 33-36]. Adenosine is believed to play a critical endogenous neuroprotectant role through effects at multiple receptors including A1, A2a, A2b, and A3. For example, adenosine, formed during the breakdown of ATP in ischemic brain regions has important anti-excitotoxic and anti-convulsant effects in brain in some model systems, while, effects of adenosine at A2a and $\mathrm{A} 2 \mathrm{~b}$ receptors can, among other effects, increase cerebral blood flow (CBF) [37, 38]. One can readily envisage that reduced metabolic demands and improved CBF represent valuable endogenous protectant effects early after brain injury from either trauma or ischemia.
We recently published two studies that provide insight into the potential neuroprotective role for adenosine in TBI. First, we reported that early after CCI, adenosine A1 receptor knockout mice develop lethal status epilepticus [36]. Status epilepticus has not been noted by us or others in numerous studies with the mouse CCI model. Nevertheless, it occurs in the A1 receptor knockout mouse even when moderate injury levels are used, and is seen independent of gender. This finding supports a powerful anti-convulsant effect of adenosine -mediated by effects at the A1 receptor-after experimental TBI, and may provided important clues toward the development of highly targeted antiexcitotoxic therapies. We have also recently reported that non-selective and A2a-selective adenosine agonists confer powerful CBF promoting effects in both the normal brain and early after CCI in rats [35] (Figure 5). It is well recognized that early after experimental and clinical TBI, CBF is reduced [39, 40]. This has suggested posttraumatic hypoperfusion or ischemia as a putative therapeutic target. Using novel perfusion MRI via the continuous arterial spinlabeling method, we demonstrated that CBF could be normalized early after injury by local injection of non-selective adenosine agonists [35]. Thus, optimal manipulation of the adenosine cascade may represent an avenue for the early post-resuscitation phase in human head injury and is an area of active investigation at our center.

\section{Serotonin 1A receptor agonists}

Another area of investigation targeting pharmacological manipulation of injured brain after severe TBI is via the serotonin transmitter system. Recent work by Dr. Anthony Kline in our center [41-43] has demonstrated beneficial effects of serotonin $1 \mathrm{~A}$ receptor agonists against both neuronal cell death and functional impairments in rats after CCI. Agents such as either repinotan or 8 hydroxy DPAT-administered after the injury-were shown to be effective [41-43]. Although the mechanism or mechanisms underlying the beneficial effects of serotonin $1 \mathrm{~A}$ receptor agonists in TBI remain to be determined, it may be more than coincidence that these agents share important anti-excitotoxic properties with adenosine and adenosine A1 receptor agonists, including the ability to hyperpolarize neurons, decrease glutamate release via actions at presynaptic glutamatergic terminals, and inhibit sodium channels. This suggests that excitotoxic pathways are of special importance early after TBI. Finally, the possibility of synergism of serotonin and 
adenosine targeting therapies in experimental or clinical TBI has not been investigated but might represent an intriguing opportunity.

\section{MRI applications in experimental TBI}

MRI is a powerful tool for the investigation of experimental TBI and cardiac arrest. We have collaborated extensively with Dr. Chien Ho and his research group at the Pittsburgh NMR center for biomedical research at Carnegie Mellon University. We have used novel perfusion MRI by the continuous arterial spin-labeling method to measure CBF in rat brain. This non-invasive method, which uses arterial water as the label, is highly advantages in that it can generate serial maps of CBF - allowing for temporal and regional assessment. In a series of studies, we have assessed $\mathrm{CO}_{2}$ reactivity after CCI [44], early posttraumatic hypoperfusion [39], the effects of anesthesia on CBF [45], serial assessment of CBF and blood-brain barrier permeability [46], study of CBF at long outcome times-as late as one year after injury [47], and the aforementioned studies of the effect of adenosine receptor agonists [33, 35]. This year, we expanded the application of this non-invasive method of CBF assessment to the studies in mice [48]. Successful adaptation of this method to the study of mice allows for the investigation of a wealth of transgenic and knockout animals, and we believe, will provide considerable insight into the contribution of posttraumatic ischemia to the evolution of secondary damage after TBI.

\section{Inflicted childhood neurotrauma}

An important problem in the field of pediatric TBI is inflicted childhood neurotrauma (child abuse, shaken baby syndrome). By assessing CSF from infants and children with severe TBI, it has become clear that victims of inflicted childhood neurotrauma often exhibit a unique biochemical profile versus victims of accidental injures (motor vehicle accidents, falls, etc). As previously discussed, victims of inflicted childhood neurotrauma may exhibit a number of unique biochemical and molecular features such as 1) a failure to mount some aspects of the acute phase response to injury such as an increase in haptoglobin levels [28],2) accentuated delayed neuronal death as reflected by a late rise in cytochrome c levels [16] and neuron specific enolase (NSE) levels [49] in CSF, and 3 ) exaggerated stress response as reflected by high CSF levels of heat shock proteins such as HSP 70 [50], among others. These findings may reflect chronic injury, delay in presentation, a propensity toward apoptosis related to the young age of these victims (they are almost always infants), and/or to the frequent occurrence of hypoxemic episodes in these patients from untreated seizures or apnea [51]. Our findings suggest that treatment of inflicted childhood neurotrauma in infants may benefit from therapies effective in hypoxic-ischemic encephalopathy (such as mild hypothermia) and possibly therapies target- ing apoptosis -which may be responsible for delayed neuronal death.

We have also measured biomarkers of brain injury (such as NSE) in serum in addition to CSF in an attempt to identify victims of mild or moderate inflicted TBI in an attempt to prevent misdiagnosis-which is an important problem for general pediatricians or emergency medicine physicians. In a recent report, Dr. Rachel Berger, working on this area at our center and Children's Hospital of Pittsburgh measured serum NSE in 98 infants who presented with non-specific symptoms that are often consistent with those seen in missed cases of inflicted childhood neurotrauma (such as vomiting without diarrhea or irritability) [52]. There was an increase in serum NSE in $79 \%$ of the children diagnosed with inflicted childhood neurotrauma and in $80 \%$ of the children who, in retrospect, may have been additional missed cases. The use of serum biomarkers, such as NSE, may represent an important new diagnostic adjunct for use in this setting [reviewed in 53].

The link between research in acute TBI and rehabilitation

Our center has traditionally focused on the acute and sub-acute phases after CNS injury-those most germane to resuscitation. However, faculty in our center in the Departments of both Neurological Surgery and Physical Medicine and Rehabilitation have been focused recently, in part, on more delayed phases after TBI. This was suggested as an important area for future research by Professor Vladimir Negovsky in his «Essays on Reanimatology» in 1980 [4]. Dixon et al [54] reported on sustained cognitive deficits in rats over a one year follow-up period after TBI produced by CCI. More recently Drs. Amy Wagner and Anthony Kline have been studying the effect of environmental enrichment on long-term cognitive outcome in rats after CCI. Environmental enrichment represents an interesting strategy that models the use of rehabilitation in the clinical setting. Environmental enrichment has powerful beneficial effects on outcome. Wagner et al [55] reported that the beneficial effect of environmental enrichment on cognitive outcome was observed only in males after CCI in rats-implying important gender differences. Subsequent studies suggest that the environmental enrichment effects on dopamine systems and neurotrophin production after experimental TBI are also, in part, gender specific [56, 57]. These studies represent on aspect of a novel approach to studying therapies in experimental TBI, namely, assessment of therapies relevant to rehabilitation in the subacute or chronic phases after injury. Both drugs and cellular therapies (such as stem cells) may have important potential in helping the brain re-wire after injury.

Cardiopulmonary arrest

Optimization of therapeutic hypothermia after cardiopulmonary arrest

A major focuses of investigation in cardiopulmonary arrest at the Safar Center is in optimizing the 


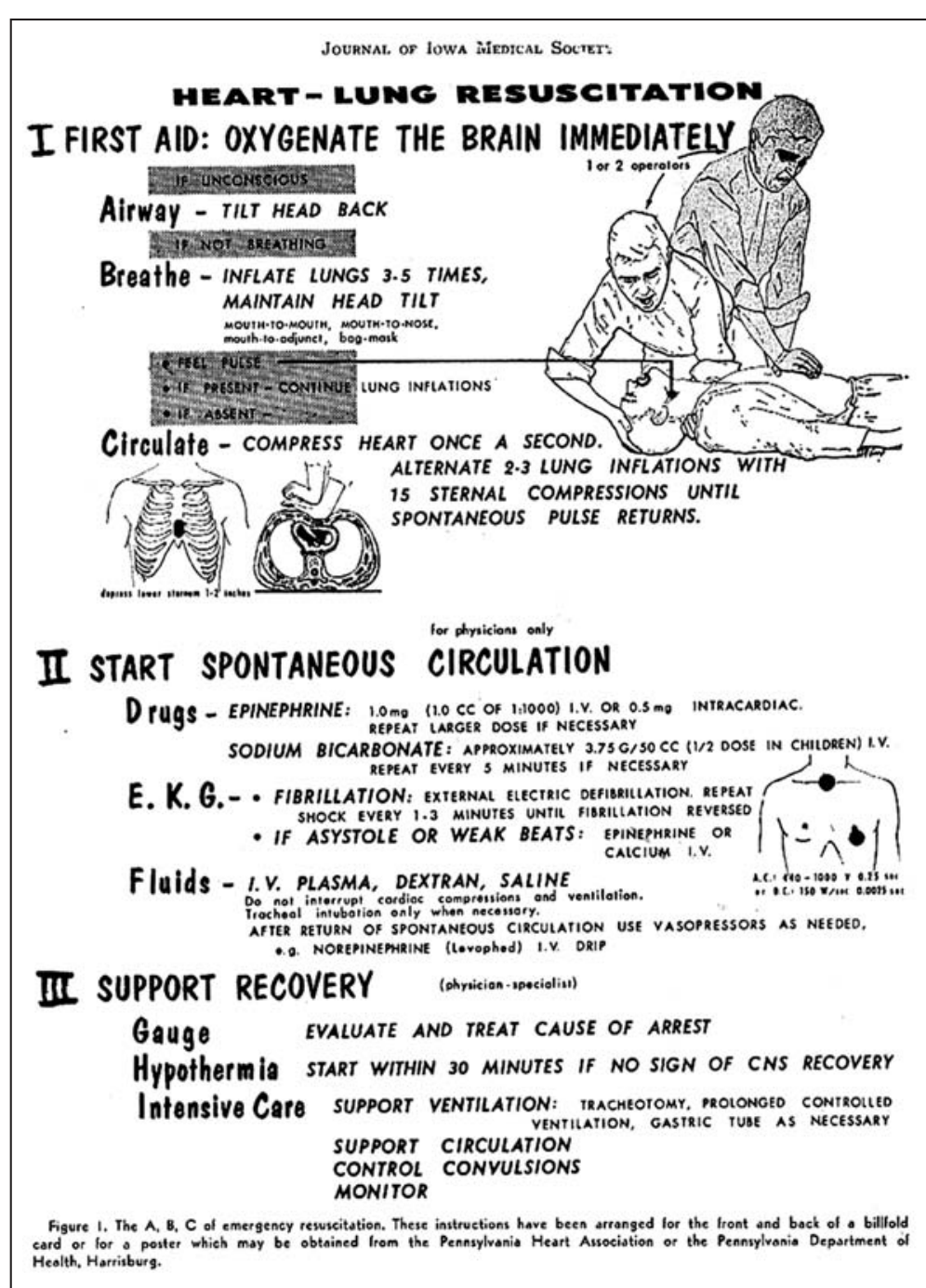

Figure 6. Initial description of the ABCs of resuscitation by Dr. Peter Safar in this classic publication in the Journal of the Iowa Medical Society. Note that Dr. Safar recommended the use of hypothermia for victims that fail to promptly recover in this early 1960s, and had already incorporated this approach into his clinical decision tree.

use of therapeutic hypothermia. Dr. Safar and a number of other investigators used of therapeutic hypothermia in the late 1950s and early 1960s. Indeed, therapeutic hypothermia was incorporated into the «ABCs» of resuscitation in the early 1960s (Figure 6) [58]. However, in that era, moderate $\left(28-32^{\circ} \mathrm{C}\right)$ rather than mild $\left(23-35^{\circ} \mathrm{C}\right)$ hypothermia was used and often for too long of a duration-leading to infections and other complications. Along with the classic paper of Busto et al [59], Dr. Peter Safar's work in clinically realistic experimental cardiac arrest launched the re-assessment of mild cooling-which has led to positive clinical trials in both adults [60,61], and newborns [62]. It is noteworthy that Dr. Fritz Sterz and his investigative team at Vienna General Hospital in Austria-an alumnus of our center-led the successful European study of mild hypothermia after cardiac arrest. These findings have led the American Heart Association and the International Liaison committee on resuscitation (ILCOR) to recommend mild cooling for comatose survivors of cardiopulmonary arrest. For more information on the use of mild hypothermia in CNS insults, we would suggest several reviews and editorials [63-65].

Recently, we have carried out two studies focused on defining the optimal application of mild hypothermia. We tested the efficacy of the application of mild hypothermia during CPR rather than after restoration of spontaneous circulation-which is the approach that was effective in the aforementioned clinical trials in both adults and infants. We used a scenario that included ventricular fibrillation (VF) followed by $3 \mathrm{~min}$ of no flow, $7 \mathrm{~min}$ of basic life support (BLS) and 10 additional min of VF with simulated unsuccessful advanced cardiac life support (ACLS). We then randomized dogs to 20 min of either continued ACLS at normothermia, or moderate hypothermia induced via venovenous extracorporeal shunt cooling. Therapeutic hypothermia induced during the arrest produced a dramatic beneficial effect-namely, normal outcomes. In contrast, dogs maintained normothermic developed multiple organ failure and remained comatose [66]. More recently, we reported that delaying the application of hypothermia from $10 \mathrm{~min}$ to $20 \mathrm{~min}$ in the resuscitation greatly reduced its effectiveness-suggesting a critical time window for the optimal intra-arrest application of cooling [67]. Coupled with the published reports on success of post-arrest cooling [60, 61] and the recent work of Bernard et al [68], our findings suggests that to optimize outcomes after cardiac arrest, cooling during resuscitation should be promptly initiated-probably with a cold intravenous bolus--and continued for 12-24 hours after resuscitation.

Asphyxial arrest-novel mechanistic insight and treatment

Related to the strong link of several of our center investigators to pediatric critical care medicine and pediatric neurointensive care, there is considerable research in the area of pediatric resuscitation. Dr. Ericka Fink, working in the laboratory of Robert Clark has developed a new rat model of asphyxial cardiopulmonary arrest mimicking the pediatric condition [69]. In that model, anesthetized rats 17 days of age are subjected to an asphyxial insult that rapidly leads to full cardiopulmonary arrest. A total insult duration of $8.5 \mathrm{~min}$ is used, and results - after resuscitation using chest compressions, epinephrine, and sodium bicarbonate administration-in survival with neuronal death in selectively vulnerable brain regions 


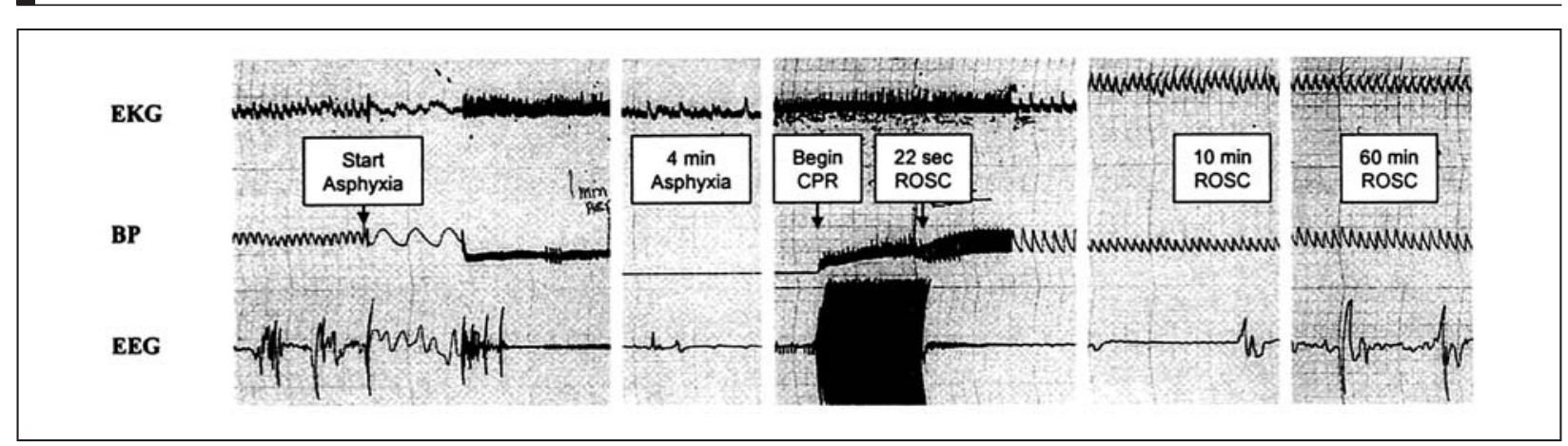

Figure 7. Electrocardiogram, blood pressure, and electroencephalogram (EEG) monitoring in a typical postnatal day 17 rat before, during, and after 8 minutes of asphyxial cardiac arrest. Partial recovery of EEG is seen after a 2-min anesthetic washout. Return of spontaneous circulation (ROSC) occurs here at 22 seconds postresuscitation. Note that the monitor speed was changed at several positions in the tracing. This model is useful for studying mechanisms and novel therapies in an experimental setting mimicking cardiopulmonary arrest in young children. CPR, cardiopulmonary resuscitation, BP, blood pressure. From Fink et al (69) with permission.

(Figure 7). This model has now been used in a series of studies to evaluate hypothermia [70] along with a number of novel therapies targeting both oxidative stress and poly-ADP ribose polymerase activation $[71,72]$. This is an area of high activity at our center and the model is an excellent one which shows great promise both for studying mechanisms and screening novel therapies.

\section{Hemorrhagic shock}

Work in the area of hemorrhagic shock in our center is under the direction of Dr. Samuel Tisherman, a trauma surgeon with critical care expertise, and is focused on novel approaches to prolong the «golden hour». The use of mild hypothermia in this regard has been evaluated in a series of studies in rats and it has been shown to powerfully delay and/or prevent acute death [73-77]. These studies have also suggested a systemic effect of hypothermia rather than a local effect on gut alone, since isolated cooling of the gut was not effective [76]. Similarly, the benefit of mild cooling does not appear to result from a reduction in cytokines [77]. One recent study from Dr. Tisherman's group is particularly noteworthy. Using a clinically relevant, pig model with trauma and intensive care, $\mathrm{Wu}$ et al [78] tested the hypothesis that mild hypothermia, induced with intravenous cold saline (ice cold or room temperature) would improve survival after hemorrhagic shock in pigs. Using a model of venous blood withdrawal over 3 hours and spleen transection, three groups were compared: 1) normothermia $\left(38^{\circ} \mathrm{C}\right)$, induced with warmed saline; 2$)$ mild hypothermia $\left(34^{\circ} \mathrm{C}\right)$ induced with intravenous infusion of iced saline; and 3 ) mild hypothermia $\left(34^{\circ} \mathrm{C}\right)$, induced with room temperature saline. After the shock phase, shed blood was returned and intensive care was continued to 24 hours. Remarkably, the hypothermia group in which cooling was achieved with room temperature saline exhibited the highest survival rate. Use of iced saline administration in shock, reduced the fluid required to achieve target blood pressure, but may appears to have maintained blood pressure at the expense of vasoconstriction and increased serum lactate levels. Thus, mild hypothermia during hemorrhagic shock, induced by infusion of room temperature saline, improves survival in a clinically relevant model of hemorrhagic shock and trauma. However, the use of iced saline in this model had detrimental effects. These findings suggest that optimal methods for induction of hypothermia must be addressed for each potential indication, e. g. cardiac arrest versus TBI versus hemorrhagic shock.

\section{(EPR)}

Emergency preservation and resuscitation

Exsanguination cardiac arrest has an extremely poor prognosis [79], likely related at least in part to the limited effectiveness of conventional resuscitation methods (i. e., CPR, BLS and ACLS) in this setting. This form of arrest is common among combat casualties and can also be seen in some cases of civilian trauma. As previously discussed, some of Dr. Negovsky's pioneering work focused on the problem of exsanguination cardiac arrest and used an intraarterial injection of oxygenated blood plus epinephrine targeting the resuscitation of exsanguinated wounded soldiers (reviewed in 3). During the last 15 years of his life, Dr. Safar worked to develop a novel approach to this otherwise refractory form of arrest. He initially termed this approach «Suspended animation for delayed resuscitation» [80]. The approach was developed in a dog model of exsanguination cardiac arrest and used a rapid aortic flush of ice cold saline (with jugular venous drainage) to promptly (within 15-20 minutes) achieve a state of profound hypothermic preservation (Figure 8). Successful emergency preservation for periods of between 1 and 3 hours would allow transport of the casualty to the far forward treatment facility in the military setting or trauma bay in the civilian setting where surgical repair could take place followed by delayed resuscitation using cardiopulmonary bypass. In a series of reports, we were able to achieve initially between $30 \mathrm{~min}$ and 1 hour of preservation andeventually -2 hours of preservation with favorable 


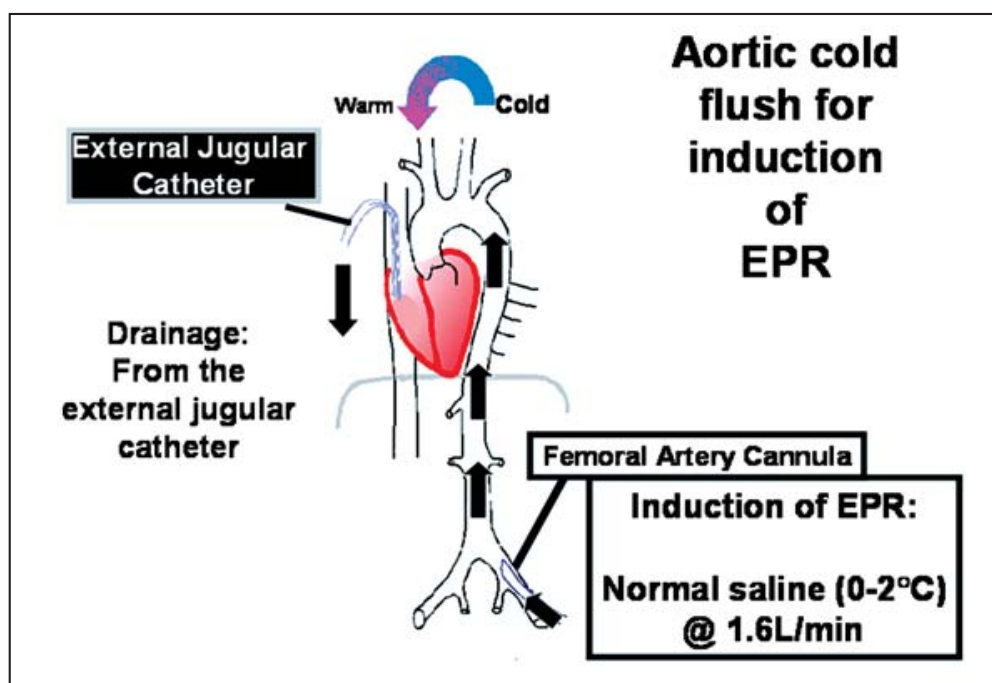

Figure 8. Schematic outlining the overall strategy used in the novel Emergency Preservation and Resuscitation (EPR) strategy recently developed at the Safar Center as a potential new approach for the treatment of exsanguination cardiac arrest. Preservation of the exsanguinated trauma victim, via rapid aortic infusion of ice-cold aortic flush (targeting a brain [tympanic] temperature of $\sim 7^{\circ} \mathrm{C}$ ), for up to 2-3 hours may allow transport to a military field surgical hospital or civilian trauma bay with surgical capability for emergent damage control surgery followed by delayed resuscitation using cardiopulmonary bypass. This approach has the potential to salvage some otherwise lethally wounded trauma victims and is ready for clinical feasibility trials.

outcome [81-83]. Some of our most recent studies suggest that 3 hours of EPR induced during exsanguination cardiac arrest may be achievable [84].

A recent publication on this topic by our center is noteworthy. Wu et al [85] tested whether or not this approach could be successful if exsanguination cardiac arrest was preceded by about 2 hours of hemorrhagic shock. In that study, good neurological outcome was achieved after a 1 hour period of hypothermic preservation even if it was preceded by 2 hours of hemorrhagic shock. Favorable outcome could only be achieved if the period of preservation was followed with 36 hours of mild hypothermia followed by slow re-warming. Dr. Drabek in our group has also recently developed a rat model of EPR to both carry out molecular studies of the mechanisms of hypothermic preservation and to screen novel therapies [86].

This novel approach to resuscitation has been named EPR in Dr. Safar's honor and may represent the new CPR for cases of refractory arrest-particularly those due to exsanguination. Finally, recent studies by Behringer in Vienna have begun to apply this approach to cases of refractory normovolemic cardiac arrest [87] and have built on Dr. Safar's suggestions of an even broader potential application of EPR. A multi-center clinical feasibility trial of EPR in the setting of civilian trauma resulting in exsanguination cardiac arrest is in the planning stages, and an initial consortium meeting of interested trauma centers was held by Dr. Tisherman in 2005 in Pittsburgh.

Conclusions and future directions

Building on the remarkable foundation laid by Dr. Peter Safar, and capitalizing on many of the important clues in the seminal work of Dr. Vladimir Negovsky, the Safar Center for Resuscitation Research continues to better understand the pathobiology of clinical conditions that require resuscitation, and ultimately improve therapies. Over the past 12 years, the scope of the work at the Safar Center has expanded to include programs in the areas of TBI and rehabilitation, and the importance of pediatric CNS injury and resuscitation have also increased in scope. Future investigation at the Safar Center, I believe, will include additional work on mechanisms in the evolution of secondary damage after TBI, cardiac arrest, and hemorrhagic shock, and development and translation of novel therapies for these conditions. New tools such as proteomics, lipidomics, genomics and advanced MRI applications are important to our ability to better understand the cybernetic post-resuscitation disease, first introduced by Dr. Negovsky, which follows these severe insults. We are also beginning to put substantial effort into studying blast injury and the combination of TBI and hemorrhagic shock. These conditions are important, albeit very unfortunate, consequences of terrorist attacks with improvised explosive devices in both the military and civilian sectors $[88,89]$. We are currently investigating novel resuscitation fluids and therapies in experimental models of these important conditions. We are also placing increased emphasis on the importance of rehabilitation, and the need for research in that area. Finally, we hope to pursue additional work in ultra-advanced resuscitation techniques, including EPR, and look forward to the challenge of an initial clinical trial in exsanguination cardiopulmonary arrest.

\section{Acknowledgement}

This article is written in honor of the late Drs. Peter Safar and Vladimir Negovsky. Dr. Kochanek is supported by the National Institutes of Health, USA, National Institute of Neurological Disorders and Stroke (NS 30318, NS 38087), the National Institute of Child Health and Human Development (T32 HD40686) the United States Army (W81XWH-06-1-0247), the Centers for Disease Control and Prevention, USA (University of Pittsburgh Center for Injury Research and Control). We also thank the Laerdal Foundation for Acute Medicine for the support of many of trainees who carried out the studies presented in this review. 


\section{Литература}

1. Safar P. From Vienna to Pittsburgh for anesthesiology and acute med icine. Careers in Anesthesiology. Autobiographical Memoirs. American Society of Anesthesiologists, Wood Library-Museum; 2000.

2. Negozsky V. A., Gurvitch A. M., Zolotokrylina E. S. Postresuscitation disease. Elsevier, Amsterdam; 1983

3. Negozsky V. A. Resuscitation and artificial hypothermia. M.: Medgiz; 1960.

4. Negozsky V. A. Essays on Reanimatology. M.: Mir Publishers; 1989.

5. Kochanek P. M., Grenvik A. A Tribute to Peter J. Safar, MD. Crit. Care Med. 2003; 31: 2571-2573.

6. Dixon C. E., Clifton G. L., Lighthall J. W. et al. A controlled cortica impact model of traumatic brain injury in the rat. J. Neurosci. Methods 1991; 39: 253-262.

7. Clark R. S. B., Kochanek P. M., Dixon C. E. et al. Early neuropathologic effects of mild or moderate hypoxemia after controlled cortical impact injury in rats. J. Neurotrauma 1997; 14: 179-189.

8. Kotapka M.J., Graham D. I., Adams J. H. et al. Hippocampal pathology in fatal human head injury without high intracranial pressure. J. Neurotrauma 1994; 11: 317-324.

9. Kochanek P. M., Clark R. S. B., Ruppel R. A. et al. Biochemical, cellular and molecular mechanisms in the evolution of secondary damage after severe traumatic brain injury in infants and children: Lessons learned from the bedside. Pediatr. Crit. Care Med. 2000; 1: 4-19.

10. Clark R. S. B., Kochanek P. M., Chen M. et al. Increases in Bcl-2 and cleavage of caspase-1 and caspase-3 in human brain after head injury FASEB. J. 1999; 13: 813-821

11. Zhang X., Graham S. H., Kochanek P. M. et al. Caspase-8 expression and proteolysis in human brain after severe head injury. FASEB J. express article 10. 1096 / fj. 02-1067 fje. Published online May 8, 2003; 17(10) 1367-1369.

12. Zhang X., Chen Y., Ikonomovic M. D. et al. Increased phosphorylation of protein kinase $B$ and its substrates after traumatic brain injury in humans and rats. J. Cereb. Blood Flow Metab. 2005 [Epub ahead of print].

13. Clark R. S. B., Kochanek P. M., Watkins S. C. et al. Caspase-3 mediated neuronal death after traumatic brain injury in rats. J. Neurochem. 2000; 74: 740-753.

14. Zhang X., Chen J., Graham S. H. et al. Mitochondrial to nuclear translocation of apoptosis-inducing factor and large scale DNA fragmentation after traumatic brain injury in rats and in neuronal cultures exposed to peroxynitrite. J. Neurochem. 2002; 82: 181-191.

15. Zhao H., Shimohata T., QangJ. Q. et al. Akt contributes to neuroprotection by hypothermia against cerebral ischemia in rats. J. Neurosci. 2005; 25: 9794-9806.

16. Satchell M. A., Lai Y., Kochanek P. M. et al. Cytochrome C, a biomarker of apoptosis, is increased in cerebrospinal fluid from infants with inflicted brain injury from child abuse. J. Cereb. Blood Flow Metab. 2005; 25: 919-927.

17. Bayir H., Kagan V. E., Tyurina Y. Y. et al. Assessment of antioxidant reserve and oxidative stress in cerebrospinal fluid after severe traumatic brain injury in infants and children. Pediatr. Res. 2002; 51: 571-578.

18. Bayir H., Kochanek P. M., Liu S. X. et al. Increased S-nitrosothiols and S-nitrosoalbumin in cerebrospinal fluid after severe traumatic brain injury in infants and children: Indirect association with intracranial pressure. J. Cereb. Blood Flow Metab. 2003; 23: 51-61.

19. Varma S., Janesko K. L., Wisniewski S. R. et al. $\mathrm{F}_{2}$-isoprostane and neuron-specific enolase in cerebrospinal fluid after severe traumatic brain injury in infants and children. J. Neurotrauma 2003; 20: 781-786.

20. Bayir H., Marion D. W., Puccio A. M. et al. Marked gender effect on lipid peroxidation after severe traumatic brain injury in adult patients. $J$. Neurotrauma 2004; 21: 1-8.

21. Bayir H., Kagan V. E., Borisenko G. et al. Enhanced oxidative stress in iNOS-deficient mice after traumatic brain injury: Support for a neuroprotective role of iNOS. J. Cereb. Blood Flow Metab. 2005; 25 673-684.

22. Bayir H., Adelson P. D., Kagan V. E. et al. Therapeutic hypothermia attenuates oxidative stress after traumatic brain injury in infants and children. 32nd SCCM Critical Care Congress, January 2003. Crit. Care Med. 2002; 30: A7.

23. Bayir H., Kagan V. E., Clark R. S. et al. Nitration and inactivation of MNSOD in brain after experimental and clinical traumatic brain injury. J. Neurotrauma. Abstr. 2005; 22: 1234

24. Bayir H., Kagan V. E., Clark R. S. B. et al. Mechanisms of tyrosine nitration of MnSOD after traumatic brain injury. Crit. Care Med. 2005; 33: A16.

25. Gopinath S. P., Robertson C. S., Contant C. F. et al. Jugular venous dsaturation and outcome after head injury. J. Neurol. Neurosurg. Psychiatry 1994; 57: 717-723.

26. Jenkins L. W., Peters G. W., Dixon C. E. et al. Conventional and functional proteomics using large format two-dimensional gel electrophoresis 24 hours after controlled cortical impact in postnatal day 17 rats. J. Neurotrauma 2002; 19: 715-740.
27. Kochanek A. R., Kline A. E., Gao W. M. et al. Gel-based proteomic analysis of rodent hippocampus 2 weeks following traumatic brain injury of immature rats using controlled cortical impact. Develop. Neurosci. (in press)

28. Gao W., Chadha M. S., Berger R. P. et al. A gel-based proteomic comparison of human cerebrospinal fluid between inflicted and non-inflicted pediatric traumatic brain injury. J. Neurotrauma (in press).

29. Clark R. S. B., Carcillo J. A., Kochanek P. M. et al. Cerebrospinal fluid adenosine concentration and uncoupling of cerebral blood flow and oxidative metabolism after severe head injury in humans. Neurosurgery 1997; 41: 1284-1293.

30. Robertson C. L., Bell M. J., Kochanek P. M. Increased adenosine in cerebrospinal fluid after severe traumatic brain injury in infants and children: Association with severity of injury and excitotoxicity. Crit. Care Med. 2001; 29: 2287-2293.

31. Bell M. J., Robertson C. S., Kochanek P. M. et al. Interstitial brain adenosine and xanthine increase during jugular venous oxygen desaturations in humans after traumatic brain injury. Crit. Care Med. 2001; 29: $399-404$.

32. Bell M.J., Kochanek P. M., CarcilloJ. A. et al. Interstitial adenosine, inosine, and hypoxanthine, are increased after experimental traumatic brain injury in the rat. J. Neurotrauma 1998; 15: 163-170.

33. Kochanek P. M., Hendrich K. S., Robertson C. L. et al. Assessment of the effect of 2-chloroadenosine on cerebral blood flow in normal rats using arterial spin labeled MRI. Magn. Reson. Med. 2001; 45: 924-929.

34. Varma M. R., Dixon C. E., Jackson E. K. et al. Administration of adenosine receptor agonists or antagonists after controlled cortical impact in mice: Effects on function and histopathology. Brain Res. 2002; 951: 191-201

35. Kochanek P. M., Hendrich K. S., Jackson E. K. et al. Characterization of the effects of adenosine receptor agonists on cerebral blood flow in uninjured and traumatically injured rat brain using continuous arterial spin-labeled magnetic resonance imaging. J. Cereb. Blood Flow Metab. 2005; 25: 1596-1612.

36. Kochanek P. M., Vagni V. A., Janesko K. L. et al. Adenosine A1 receptor knockout mice develop lethal status epilepticus after experimental traumatic brain injury. J. Cereb. Blood Flow Metab. 2006; 26: 565-575.

37. Miller L. P., Hsu C. Therapeutic potential for adenosine receptor activation in ischemic brain injury. J. Neurotrauma 1992; 9: S563-S577.

38. Fredholm B. B. Adenosine receptors as targets for drug development. Drug News Perspect. 2003; 16: 283-289.

39. Hendrich K., Schiding J., Kochanek P. et al. Early perfusion after controlled cortical impact in rats: Quantification by arterial spin-labeled MRI and the influence of spin-lattice relaxation time heterogeneity. Magn. Reson. Med. 1999; 42: 673-681.

40. Marion D. W., Darby J., Yonas H. Acute regional cerebral blood flow changes caused by severe head injuries. J. Neurosurg. 1991; 74: 407-414.

41. Kline A. E., Yu J., Horvath E. et al. The selective 5-HT(1A) receptor agonist repinotan HC1 attenuates histopathology and spatial learning deficits following traumatic brain injury in rats. Neurosci. 2001; 106: 547-555.

42. Kline A. E., Yu J., Massucci J. L. et al. Protective effects of the 5-HT1A receptor agonist 8-hydroxy-2-(di-n-propylamino)tetralin against traumatic brain injury-induced cognitive deficits and neuropathology in adult male rats. Neurosci. Letters 2002; 333: 179-182.

43. Kline A. E., Massucci J. L., Dixon C. E. et al. The therapeutic efficacy conferred by the 5-HT(1A) receptor agonist 8-Hydroxy-2-(di-n-propylamino)tetralin (8-OH-DPAT) after experimental traumatic brain injury is not mediated by concomitant hypothermia. J. Neurotrauma 2004; 21: 175-185.

44. Forbes M. L., Hendrich K. S., Kochanek P. M. et al. Assessment of cerebral blood flow and $\mathrm{CO}_{2}$ reactivity after controlled cortical impact by perfusion magnetic resonance imaging using arterial spin labeling in rats. J. Cereb. Blood Flow Metab. 1997; 17: 865-874

45. Hendrich K. S., Kochanek P. M., MelickJ. A. et al. Cerebral perfusion during anesthesia with fentanyl isoflurane or pentobarbital in normal rats studied by arterial spin-labeled MRI. Magn. Reson. Med. 2001; 46: 202-206.

46. Hendrich K., SchidingJ., Kochanek P. et al. Sequential MRI assessment of cerebral blood flow and blood-brain barrier permeability early after traumatic brain injury in rats. J. Cereb. Blood Flow Metab. 1997; 17: S76.

47. Kochanek P. M., Hendrich K. S., Dixon C. E. et al. Cerebral blood flow at one year after controlled cortical impact in rats: Assessment by magnetic resonance imaging. J. Neurotrauma 2002; 19: 1029-1037.

48. Foley L. M., Hitchens T. K., Kochanek P. M. et al. Murine orthostatic response during prolonged vertical studies: Effect on cerebral blood flow measured by arterial spin-labeled MRI. Magn. Reson. Med. 2005; 54: 798-806.

49. Berger R. P., Janesko K. L., Wisniewski S. R. et al. Neuron-specific enolase and S100B in cerebrospinal fluid after severe traumatic brain injury in infants and children. Pediatrics 2002; 109(2), URL: http: //www. pediatrics. org/cgi/content/full/109/2/e31.

50. Lai Y. C., Stange C., Wisnieroski S. R. et al. Mitochondrial heat shock protein 60 is increased in cerebrospinal fluid following pediatric traumatic brain injury. Dev. Neurosci. (in press). 
51. Berger R. P., Adelson P. D., Richichi R. et al. Serum biomarkers after traumatic and hypoxemic brain injuries: Insight into the biochemical response of the pediatric brain to inflicted brain injury. Dev. Neurosci. (in press).

52. Berger R. P., Dulani T., Adelson P. D. et al. Identification of brain injury in well-appearing infants using serum and cerebrospinal markers: A possible screening tool. Pediatrics 2006; 117: 325-332.

53. Berger R. P., Kochanek P. M., Pierce M. C. Biochemical markers of brain injury: Could they be used as diagnostic adjuncts in cases of inflicted traumatic brain injury? (Invited Review) Child Abuse and Neglect: Int. J. 2004; 28: 739-754.

54. Dixon C. E., Kochanek P. M., Yan H. Q. et al. A One-year study of spatial memory performance, brain morphology and cholinergic markers after moderate controlled cortical impact in rats. J. Neurotrauma 1999; 16 : 109-122.

55. Wagner A. K., Kline A. E., Sokoloski J. et al. Intervention with environmental enrichment after experimental brain trauma enhances cognitive recovery in male but not female rats. Neurosci. Letters 2002; 334: $165-168$.

56. Chen X., Li Y., Kline A. E. et al. Gender and environmental effects on regional brain-derived neurotrophic factor expression after experimental traumatic brain injury. Neuroscience $2005 ; 135$ : 11-17.

57. Wagner A. K., Chen X., Kline A. E. et al. Gender and environmental enrichment impact dopamine transporter expression after experimental traumatic brain injury. Exp. Neurol. 2005; 195: 475-483

58. Safar P. Community-wide cardiopulmonary resuscitation. J. Iowa Med. Soc. $1964 ; 629-635$.

59. Busto R., Dietrich W. D., Globus M. Y. et al. Small differences in intraischemic brain temperature critically determine the extent of ischemic neuronal injury. J. Cereb. Blood Flow Metab. 1987; 7: 729-738.

60. Bernard S. A., Gray T. W., Buist M. D. et al. Treatment of comatose survivors of out-of-hospital cardiac arrest with induced hypothermia. N. Engl. J. Med. 2002; 346: 557-563.

61. Hypothermia after cardiac arrest study group: mild therapeutic hypothermia to improve the neurologic outcome after cardiac arrest. $\mathrm{N}$. Engl. J. Med. 2002; 346: 549-556.

62. Shankaran S., Laptook A. R., Ehrenkranz R. A. et al. National institute of child health and human development neonatal research network: whole-body hypothermia for neonates with hypoxic-ischemic encephalopathy. N. Engl. J. Med. 2006; 353: 1574-1584.

63. Safar P. J., Kochanek P. M. Resuscitative hypothermia after cardiac arrest. Invited editorial, N. Engl. J. Med. 2002; 346: 612-613.

64. Kochanek P. M., Safar P.J. Therapeutic hypothermia for severe traumatic brain injury. Invited editorial, J. Amer. Med. Assoc. 2003; 289: 3007-3009

65. Kochanek P. M. Brain Trauma: Laboratory Studies. In: Tisherman S. A., Sterz F. (eds. ) Therapeutic hypothermia. Springer Science +Business Media, Inc. 2005. 63-86.

66. Nozari A., Safar P., Stezoski S. W. et al. Mild hypothermia during prolonged cardiopulmonary-cerebral resuscitation increases conscious survival in dogs. Crit. Care Med. 2004; 32: 2110-2116. (Accompanying editorial) 2004; 32: 2164-2165.

67. Nozari A., Safar P., Stezoski S. W. et al. Critical time window for the induction of mild hypothermia during cardiopulmonary resuscitation from prolonged circulatory arrest in dogs. Circulation (in press).

68. Bernard S., Buist M., Monteiro O., Smith K. Induced hypothermia using large volume, ice-cold intravenous fluid in comatose survivors of outof-hospital cardiac arrest: a preliminary report. Resuscitation 2003; 56: $9-13$.

69. Fink E. L., Alexander H., Marco C. D. et al. Experimental model of pediatric asphyxial cardiopulmonary arrest in rats. Pediatr. Crit. Care Med. 2004; 5: 139-144.

70. Fink E. L., Marco C. D., Donovan H. A. et al. Brief induced hypothermia improves outcome in a pediatric model of asphyxial cardiopulmonary arrest in rats. American stroke association; fellows' research day, Hilton hotel. Pittsburgh, PA, February 13, 2004.

71. Fink E. L., Bayir H., Lai Y. et al. Clark RS: $\gamma$-glutamylcysteine ethyl ester increases brain glutathione after asphyxial cardiac arrest in post-natal day 17 rats: 86. 34th SCCM Critical care congress, Crit. Care Med. 2004; 32(12 Suppl): A22.

72. Fink E. L., Marco C. D., Chen Y. et al. Protracted increase in hippocampal Poly-ADP-ribosylation after asphyxial cardiac arrest in juvenile rats. Pediatric academic societies' annual meeting; 2005; PAS 57: 51.

73. Kim S. H., Stezoski S. W., Safar P. et al. Hypothermia and minimal fluid resuscitation increase survival after uncontrolled hemorrhagic shock in rats. J. Trauma 1997; 42: 213-222.

74. Kim S. H., Stezoski S. W., Safar P. et al. Hypothermia, but not $100 \%$ oxygen breathing, prolongs survival time during lethal uncontrolled hemorrhagic shock in rats. J. Trauma 1998; 44: 485-491.

75. Takasu A., Carrillo P., Stezoski S. W. et al. Mild or moderate hypothermia but not increased oxygen breathing prolongs survival during letha uncontrolled hemorrhagic shock in rats, with monitoring of visceral dysoxia. Crit. Care Med. 1999; 27: 1557-1564.

76. Wu X., Stezoski J., Safar P., Behringer W. et al. Systemic hypothermia, but not regional gut hypothermia, improves survival from prolonged hemorrhagic shock in rats. J. Trauma 2002; 53: 654-662.

77. Wu X., Stezoski J., Safar P. et al. Mild hypothermia during hemorrhagic shock in rats improves survival without significant effects on inflammatory responses. Crit. Care Med. 2003; 31: 195-202.

78. Wu X., Kochanek P., Cochran K. et al. Mild hypothermia improves survival after prolonged, traumatic hemorrhagic shock in pigs. J. Trauma 2005; 59: 291-299; discussion 299-301.

79. Mullie A., Lewi P., Van Hoeyweghen R. Pre-CPR conditions and the final outcome of CPR. The cerebral resuscitation study group. Resuscitation 1989; 17(Suppl): S11-S21.

80. Bellamy R., Safar P., Tisherman S. A. et al. Suspended animation for delayed resuscitation. Crit. Care Med. 1996; 24 (2 Suppl): S24-S47.

81. Behringer W., Prueckner S., Kentner R. et al. Rapid hypothermic aortic flush can achieve survival without brain damage after 30 minutes cardiac arrest in dogs. Anesthesiology 2000; 93: 1491-1499.

82. Behringer $W$., Safar $P$., Wu X. et al. Survival without brain damage after clinical death of $60-120$ mins in dogs using suspended animation by profound hypothermia. Crit. Care Med. 2003; 31: 1523-1531.

83. Nozari A., Safar P., Wu X. et al. Suspended animation can allow survival without brain damage after traumatic exsanguination cardiac arrest of 60 minutes in dogs. J. Trauma 2004; 57: 1266-1275.

84. Wu X., Drabek T., Tisherman S. A. et al. Emergency preservation and resuscitation with energy substrates and hypothermia allow reliable neurological recovery after $3 \mathrm{~h}$ of cardiac arrest following rapid exsanguination in dogs. International Anesthesia Research Society Control No 1357 , (in press).

85. Wu X., Kochanek P. M., Drabek T. et al. Induction of profound hypothermia for emergency preservation and resuscitation allows intact survival from cardiac arrest resulting from prolonged lethal hemorrhage and trauma in dogs. Circulation 2006; 113: 1974-1982.

86. Drabek T., Stezoski J., Wu X. et al. Establishment of a rat model of suspended animation with delayed resuscitation: A preliminary report: 211. Crit. Care Med. 2004; 32(12 Suppl): A56.

87. Janata A., Holzer M., Bayegan K. et al. Rapid induction of cerebral hypothermia by aortic flush during normovolemic cardiac arrest in pigs. Crit. Care Med. 2006; 34: 1769-1774.

88. DePalma R. G., Burris D. G., Champion H. R. et al. Blast injuries. N. Engl. J. Med. 2005; 352: 1335-1342.

89. Okie S. Traumatic brain injury in the war zone. N. Engl. J. Med. 2005; 352: 2043-2047.

Поступила 20. 06. 06 